\title{
THE INDUCTION OF AN ATP-ENERGIZED MITOCHONDRIAL VOLUME CHANGE BY THE COMBINATION OF THE -S-S- COMPOUND GLIOTOXIN WITH EITHER A RESPIRATORY INHIBITOR OR AN UNCOUPLING AGENT*********
}

\author{
Herbert I. Hadler, Mitcheld R. Hadler \\ and Barbara. G. Daniel \\ Department of Chemistry and Biochemistry, \\ Southern I11inois University, Carbondale, I11. 62901, U.S.A. \\ (Received for publication October 12, 1972)

\begin{abstract}
When gliotoxin, an antibiotic, an antitumor agent and an antiviral agent, was combined with either a respiratory inhibitor or an uncoupling agent, an ATP-energized mitochondrial volume change was induced. The effect was attributed to the reaction of an exposed nucleophilic mitochondrial thiol group with the electrophilic -S-S- group in gliotoxin. While this type of interaction may be applied to assist the antitumor and toxic activities of gliotoxin it is difficult to relate the reported interaction to the antiviral activity of gliotoxin except as a model reaction.
\end{abstract}

In a previous report ${ }^{1}$ ) we observed that ELLMAN's reagent (a thiol reagent which possesses the -S-S- group) when combined with either a respiratory inhibitor such as antimycin or an uncoupling agent such as DNP induced an ATP-energized mitochondrial volume change. According to the rationalization which we have used on many previous occasions $2^{2,3,4,5)}$ it follows that ELLMAN's reagent became conjugated with a mitochondrial thiol group which was exposed either by the respiratory inhibitor or the uncoupling agent. This activity of Ellman's reagent raised the question whether other compounds of biological interest which also have the disulfide function could interact with the mitochondrial thiol group exposed either by a respiratory inhibitor or an uncoupling agent. We have accordingly examined the role of gliotoxin in this phenomenon. Such an interaction would be visualized by an appropriate ATP-energized mitochondrial volume change. Gliotoxin is of biological interest because it is an antibiotic, ${ }^{6)}$ an antitumor agent, ${ }^{6)}$ and one of the few agents known to have antiviral activity. ${ }^{7,8}$

* Supported by U.S.P.H.S. grant CA 10759 (National Cancer Institute).

** Abbreviations used :

ATP : Adenosine- $5^{\prime}$-triphosphate

ADP : Adenosine-5'-diphosphate

$\mathrm{Pi}$ : Inorganic phosphate

DNP : 2,4-Dinitrophenol

NEMI : N-Ethylmaleimide

Di-OH-DBAQ : $4^{\prime}, 8^{\prime}$-Dihydroxy-1,2,5,6-dibenz-9,10-anthraquinone

*** Gliotoxin is $1 \mathrm{OH}-3,10 \mathrm{a}$-epidithiopyrazino $[1,2$-a]indole-1, 4-dione-2, 3, 5a, 6-tetrahydro-6-hydroxy-3 (hydroxymethyl)-2-methyl. CA registry number is 67-99-2. 


\section{Methods}

General procedures, methods, and purification of the water have been previously described ${ }^{9)}$, for the mitochondrial volume-change experiments. The $\mathrm{pH}$ of the Tris-chloride buffer is indicated on the charts. Incubation was at $27^{\circ} \mathrm{C}$ in standard rectangular glass cuvettes with a $1-\mathrm{cm}$ light path. The basic reaction mixture for the volume change experiments had a final volume of $3 \mathrm{ml}$ and contained $0.75 \mathrm{mg}$ mitochondrial protein (prepared from rat liver), $75 \mathrm{~mm}$ sucrose, and $75 \mathrm{~mm}$ Trischloride buffer. A decrease in absorbance at $520 \mathrm{~nm}$ was taken as a measure of mitochondrial swelling. A Model 2000 automatic spectrophotometer manufactured by Gilford Instrument Laboratories, Inc., Oberlin, Ohio, was used. All cations were added in the form of chloride salts, and anions were added in

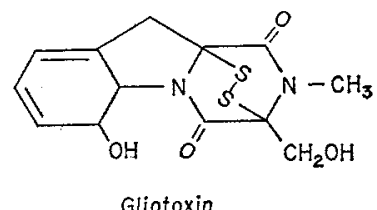

Gliotoxin the form of Tris salts neutralized to $\mathrm{pH}$ 7.4. Solutions of gliotoxin, antimycin and oligomycin were prepared in $95 \%$ ethanol (distilled). All controls contained the appropriate amount of $95 \%$ ethanol. A cuvette never contained more than $0.06 \mathrm{ml}$ of $95 \%$ ethanol. The adding-mixing device was used in making additions after the mitochondria were added to the cuvette. The charts and legends provide additional experimental details.

Samples of gliotoxin were generously provided by Dr. P. W. Trown, Lederle Laboratories, American Cyanamid Co., Pearl River, New York 10965, U.S.A. and by Dr. N.M. Larin, Pfizer Ltd., Ramsgate Road, Sandwich, Kent, England.

\section{Results}

Neither antimycin nor gliotoxin by themselves induced an ATP-energized mitochondrial volume change phenomenon. The combination of these two reagents did induce an ATP-energized mitochondrial volume change (Fig. 1). There was a considerable lag period before the effects induced by the two agents became apparent.

Fig. 1. Gliotoxin with antimycin.

Basic medium see methods. The cuvette contained ATP and antimycin prior to the addition of mitochondria. Gliotoxin dissolved in $95 \%$ ethanol or $95 \%$ ethanol was added after the addition of mitochondria.
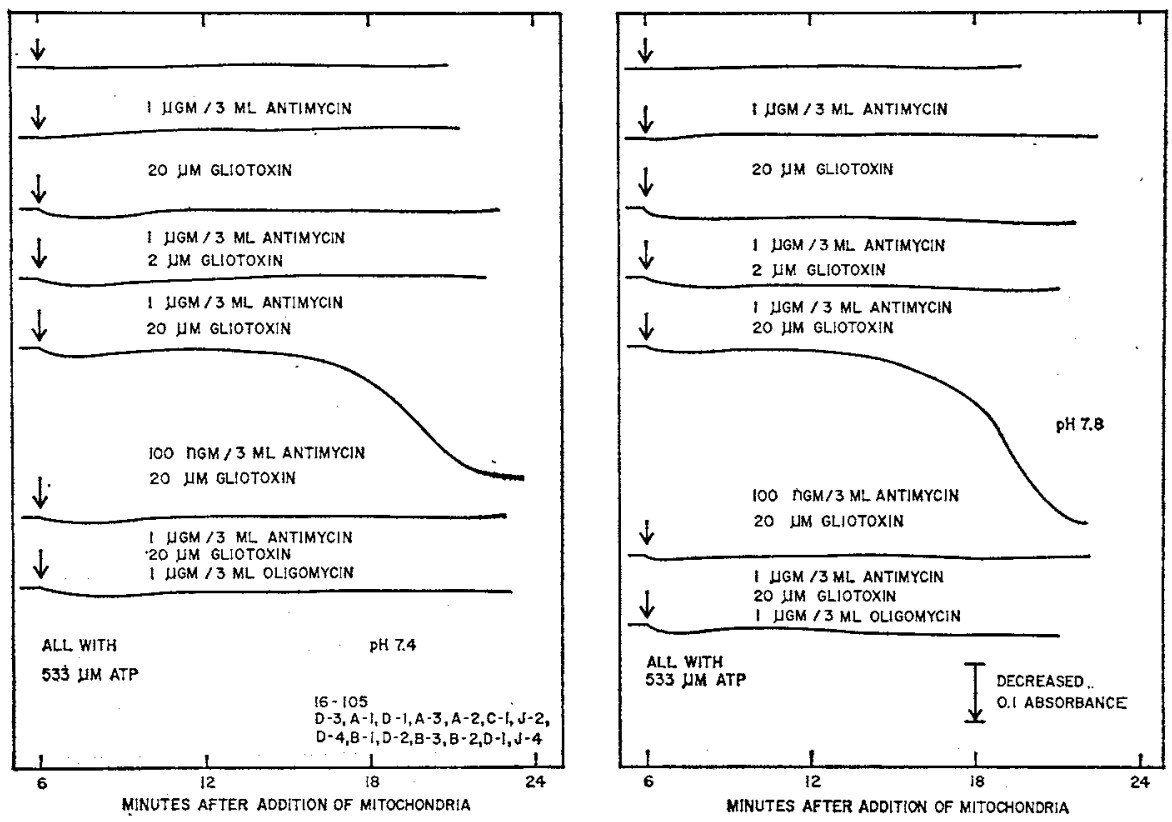
Fig. 2. Gliotoxin with DNP.

Basic medium see methods. The cuvette contained ATP prior to the addition of mitochondria. Following the addition of mitochondria, gliotoxin dissolved in $95 \%$ ethanol or $95 \%$ ethanol was added followed by DNP.
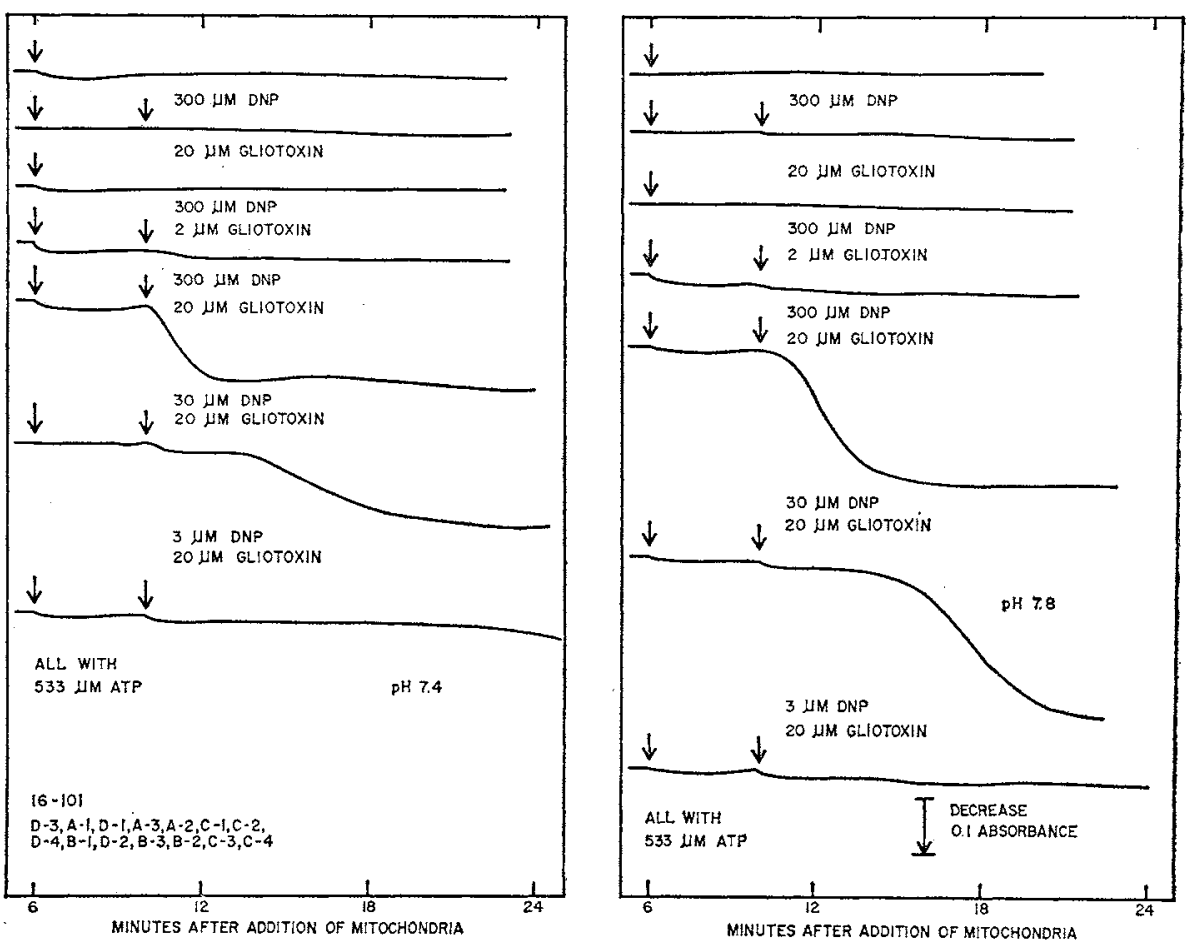

Fig. 3. Oligomycin and gliotoxin combined with DNP.

Basic medium see methods. The cuvette contained ATP and oligomycin prior to the addition of mitochondria. The first addition after mitochondria was gliotoxin dissolved in $95 \%$ ethanol or $95 \%$ ethanol. The second addition after mitochondria was DNP.
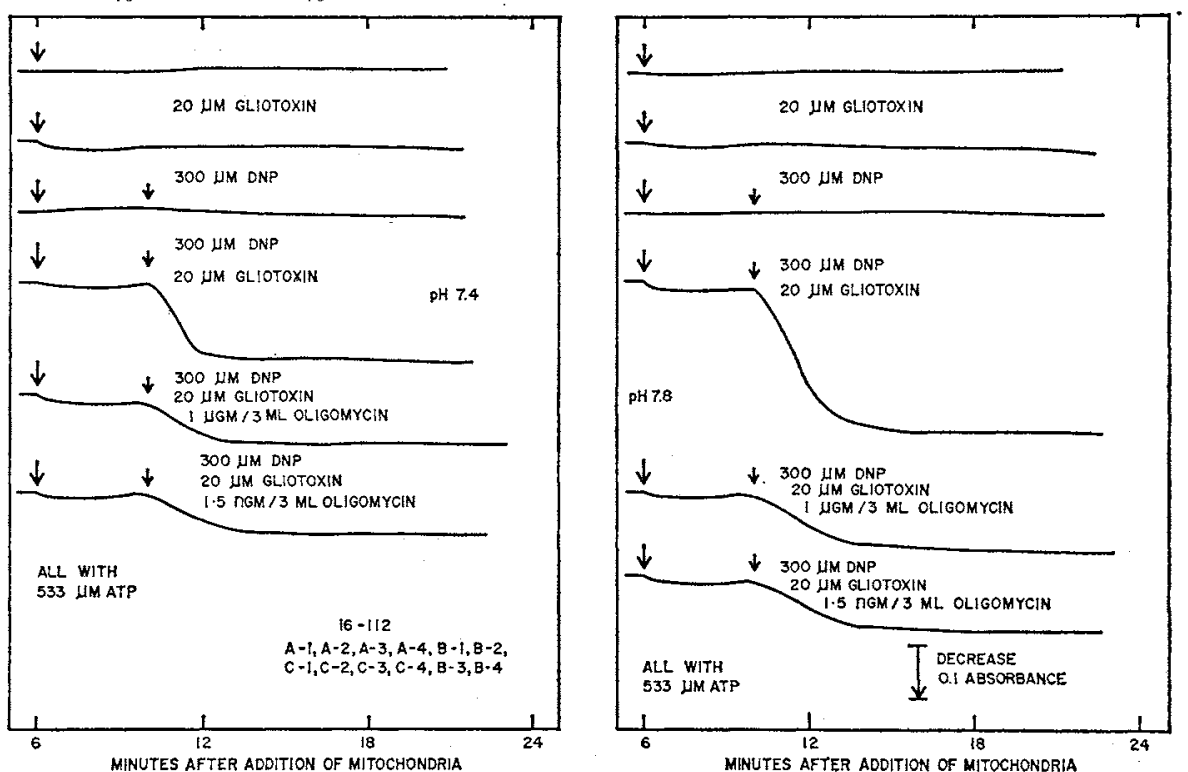
Fig. 4. Role of ions with gliotoxin and DNP.

Basic medium see methods. The cuvette contained ATP prior to the addition of mitochondria. The order of additions after mitochondria were firstly, gliotoxin in $95 \%$ ethanol or $95 \%$ ethanol and secondly DNP.
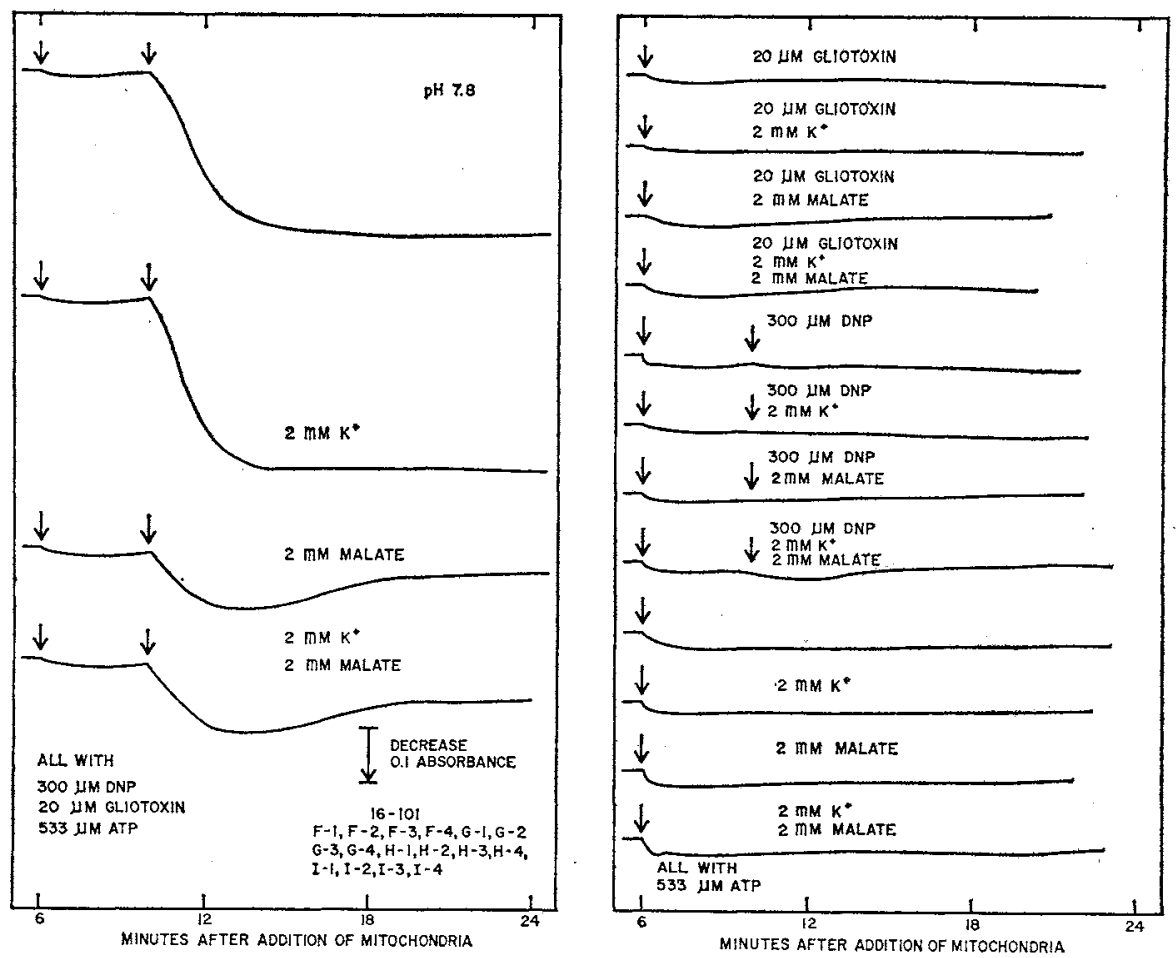

It should be noted, however, that in our previous experiments the thiol reagent, [showdomycin, ${ }^{2,3)}$ NEMI, ${ }^{5}$ and ELLMAN's reagent ${ }^{1)}$ ] were incubated with mitochondria for several minutes before ATP was added, while in these experiments gliotoxin was added to the cuvette after the mitochondria, and gliotoxin also was the final component of the system. Thus the delay in action may be ascribed to a requirement for time so that gliotoxin may penetrate the mitochondria and reach the appropriate reactive site. It also is seen in Fig. 1 that adequate levels of gliotoxin [20 $\mu \mathrm{M}]$ and antimycin [1 $\mu g$ per $3 \mathrm{ml}]$ were needed. When the $\mathrm{pH}$ was raised, the effect was enhanced. The induced effect was inhibited completely by oligomycin.

In Fig. 2 it is seen that while neither DNP nor gliotoxin by themselves induced an ATP-energized mitochondrial volume change, a combination of these two agents did induce an ATP-energized mitochondrial volume change. Attention should be directed at the conditions which are different than those employed in the previous experiments with antimycin. If $\mathrm{ATP}$-had been exposed simultaneously to mitochondria and DNP prior to the addition of gliotoxin the induced ATPase activity would have depleted the ATP-and there would be no source of chemical potential to drive the ATP-energized mitochondrial volume change when the system was completed by the addition of gliotoxin. It was thus reasonable to add DNP as the final component. As gliotoxin was a water-insoluble compound, it was necessary to add gliotoxin after the mitochondria. The order of additions to the cuvette was therefore, mitochondria, 
gliotoxin, and DNP. Under these conditions a prompt ATP-energized mitochondrial volume change was induced by the combination of DNP and gliotoxin. The effect was enhanced when the $\mathrm{pH}$ was raised. The effect required adequate levels of gliotoxin $[20 \mu \mathrm{M}]$ and DNP $[300 \mu \mathrm{M}]$.

It is seen in Fig. 3 that the prompt ATP-energized mitochondrial volume change induced by the combination of DNP plus gliotoxin was decidedly inhibited by oligomycin. However, the inhibition was not complete, and in the situation where the induced effects had been enhanced by raising the $\mathrm{pH}$, the inhibition was somewhat less complete. This result is reminiscent of the incipient lack of inhibition by oligomycin, noted previously, ${ }^{1)}$ on the effect induced by the combination of antimycin or DNP plus ELLMAN's reagent, at pH 7.8 and $300 \mu \mathrm{M}$ ELLmaN's reagent.

In Fig. 4, it is seen that the phenomenon induced by the combination of DNP plus gliotoxin was not affected by the addition of potassium ion but was markedly reduced by addition of malate ion.

\section{Discussion}

Gliotoxin by itself did not induce an ATP-energized mitochondrial volume change. However, when gliotoxin was combined with either antimycin or DNP an ATP-energized mitochondrial volume change was induced. The phenomenon was enhanced by increasing the concentration of gliotoxin and by raising the $\mathrm{pH}$ and was dependent on an adequate level of antimycin or DNP. The phenomenon was decidedly inhibited but not completely inhibited by oligomycin. Thus there is a partial by-pass of the oligomycin-sensitive site in the presence of gliotoxin. This by-pass was only incipient with Eliman's reagent. All these data are in agreement with the rationalization that gliotoxin participates as an electrophilic thiol reagent in an interaction with a nucleophilic mitochondrial thiol group, exposed either by a respiratory inhibitor or an uncoupling agent. Because of the insolubility of gliotoxin it was necessary to use special conditions previously employed with DI-OHDBAQ.9) Under these conditions gliotoxin was added subsequent to the addition of mitochondria so that the freshly dispersed gliotoxin would be taken up by the mitochondria before the dispersed gliotoxin had an opportunity to settle out. While potassium ion had no effect there was a decided effect due to malate ion. Malate ion decidedly minimized the ATP-energized mitochondrial volume change. We have never rationalized the effect of malate ion in similar ATP-energized mitochondrial volume change systems. We have observed that malate ion has various effects. The effect has not been identical from system to system and depends upon the conditions employed $d^{2,3,4,5)}$. Potassium ion has not had any effect on these systems thus far. ${ }^{1,2,3,4,5,9)}$ It is conceivable that the role of malate ion depends on the extent of damage to the mitochondria and whether malate ion in some instances serves as a permeant anion or malate ion competes with another permeant anion such as phosphate which may have leaked out of the mitochondria. However the exact role of malate ion is at present unknown.

The interaction of gliotoxin with the exposed pivotal mitochondrial group does suggest a possible role for gliotoxin as an antitumor agent. We have previously pointed out that the combinations of agents which interact cooperatively with mitochondria might enhance the tumor inhibiting properties of the individual agents. ${ }^{10)}$ It would thus be reasonable to examine the combination of gliotoxin with an antitumor agent which is either a respiratory inhibitor or an uncoupling agent (e.g. lapachol).

Based upon the current understanding of viral replication it is difficult to relate the antiviral activity of gliotoxin to the phenomena observed here. However, the mode of interaction of gliotoxin with a mitochondrial thiol group may be a model for a reaction 
responsible for the inhibition of viral replication by gliotoxin. Trown ${ }^{11}$ studied the antiviral activity of gliotoxin and other compounds with the epidithiapiperazinedione moiety demonstrated a requirement for the $-\mathrm{S}-\mathrm{S}$ - grouping in the compounds which were active antiviral agents.

BREWer et al. ${ }^{12)}$ and BREWER and $T_{A Y L O R}{ }^{13)}$ ascribed the toxic properties of gliotoxin and other epidithiapiperazinediones to the $-\mathrm{S}-\mathrm{S}$ - functional group. As gliotoxin was toxic without the assistance of a respiratory inhibitor or an uncoupling agent these biological results are not readily explained by the participation of gliotoxin in an ATP-energized mitochondrial volume change which also requires the presence of either a respiratory in hibitor or an uncoupling agent.

\section{References}

1) Hadler, H. I.; M. R. Hadler \& B. G. Daniel: The induction of an ATP-energized mitochondrial volume change by the combination of the -S-S- compound, Eluman's reagent with either a respiratory inhibitor or an uncoupling agent. J. Antibiotics $26: 23 \sim 29,1973$

2) Hadier, H. I.; B. E. Claybourn \& T.P. Tschang: Mitochondrial volume changes induced by the antibiotic showdomycin. Biochem. Biophys. Res. Commun. $31: 25 \sim 31,1968$

3) Haduer, H. I.; B. E. Claybourn \& T. P. Tschang: The mode of action of dinitrophenol revealed by mitochondrial volume changes requiring rotenone or antimycin or dinitrophenol with showdomycin. J. Antibiotics $21: 575 \sim 581,1968$

4) Hadler, H. I.; B. E. Claybourn, T. P. Tschang \& T. L. Moreau : The pivotal position of the mitochondrial thiol group exposed by dinitrophenol located by means of ATP energized mitochondrial volume changes requiring gramicidin, showdomycin, and dinitrophenol. J. Antibiotics $22: 183 \sim 188,1969$

5) Hadler, H. I.; B. E. Claybourn \& T.P. Tschang: Combinations of agents which induce ATP energized mitochondrial volume changes. J. Antibiotics $23: 276 \sim 287,1970$

6) Spector, W. S. : Handbook of Toxicology. Saunder, Philadelphia 1957, Vol. 2, p. 84, 220

7) Rightseld, W. A.; H. G. Schneider, B.J. Sloan, P.R. Graf, F.A. Miller, Q.R. Bartz, J. Ehrlich \& G. J. Dixon: Antiviral activity of gliotoxin and gliotoxin acetate. Nature $204: 1333 \sim 1334$, 1964

8) Larin, N. M.; M. P. Copping, R. H. Herbst-Laier, B. Roberts \& R.B.M. Wenham : Antiviral activity of gliotoxin. Chemotherapia $10: 12 \sim 23,1965$

9) Hadler, H. I.; B. G. Daniel, J. Demetriou \& R. C. Pratt : The induction of ATP energized mitochondrial volume changes by showdomycin when combined with $4^{\prime}, 8^{\prime}$-dihydroxy-1,2,5,6dibenz-9,10-anthraquinone, a metabolite of the carcinogenic polynuclear hydrocarbon dibenz(a, h) anthracene. J. Antibiotics $24: 835 \sim 845,1971$

10) Hadler, H. I. \& T. L. Moreau : The induction of ATP energized mitochondrial volume changes by the combination of the two antitumor agents showdomycin and lapachol. J. Antibiotics $22: 513 \sim 520,1969$

.11) Trown, P.W.: Antiviral activity of $N, N^{\prime}$-dimethyl-epidithia-piperazinedione, a synthetic compound related to the gliotoxins, LL-S8 $\alpha$ and $\beta$, chetomin and sporidesmins. Biochem. Biophys. Res. Commun. $33: 402 \sim 407,1968$

12) Brewer, D.; D. E. Hannah \& A. Taylor: The biological properties of 3,6-epidithiadiketopiperazines. Inhibition of growth of Bacillus subtilis by gliotoxins, sporidesmins and chetomin. Can. J. Microbiol. $12: 1187 \sim 1195,1966$

13) Brewer, D. \& A. TAYLOR: The biological properties of 3,6-epidithiadiketopiperazines. Degradation of gliotoxin-B by Bacillus subtilis (HLX 373). Can. J. Microbiol. 13: 1577 1589, 1967 